\title{
CROIX-ROUGE, CROISSANT-ROUGE ET COMMUNICATION
}

Le monde contemporain vit un gigantesque paradoxe. On $y$ constate une interdépendance accrue des sociétés humaines et des problèmes qui se posent à l'homme, mais en même temps la plus grande diversité caractérise ces sociétés, qu'elle se traduise par les écarts croissants dans le développement des pays ou qu'elle résulte des aspirations des individus et des communautés à affirmer leur identité. L'enjeu en cette fin $d u X X^{e}$ siècle est de créer une véritable solidarité humaine qui atténuerait les disparités et les inégalités tout en respectant les différences et les identités.

Emanation de toute société, la communication conçue comme relation d'individu à individu, ou comme moyen d'informer et d'éduquer, $a$ un rôle capital à jouer non seulement pour propager le savoir, permettre aux communautés de mieux se comprendre et par là même diminuer les tensions, mais aussi pour faciliter l'expression libre de personne à personne, de groupes à groupes, et sauvegarder l'intégrité culturelle des uns et des autres.

Transposées au sein du Mouvement international de la CroixRouge et du Croissant-Rouge, ces idées montrent qu'il existe depuis longtemps une véritable "solidarité d'image" entre ses diverses composantes et qu'au delà des différences idéologiques et culturelles inhérentes à un mouvement universel, s'impose une identité, une spécificité du Mouvement qu'inspirent ses Principes fondamentaux et sa mission humanitaire.

Mais il ne suffit pas de constater l'évidence de la solidarité humaine ni de s'enorgueillir passivement de la spécificité du Mouvement, encore faut-il pouvoir les entretenir, les imposer aux yeux du public, encore faut-il savoir présenter le Mouvement dans sa globalité tout en s'efforçant de développer les moyens de promouvoir ses principes et son action.

D'où l'adoption par le Conseil des Délégués, en octobre 1989, d'une politique d'information dont le but est de renforcer l'aptitude du Mouvement à communiquer, sur les plans international et national, une image claire et cohérente de sa mission, et par là de développer 
sa capacité à accomplir ses tâches et d'accroître le soutien du public.

Aussi la Revue internationale de la Croix-Rouge a-t-elle jugé utile d'ouvrir, avec le présent numéro, un dossier sur la genèse de la politique d'information du Mouvement, sur ses tenants et aboutissants qui concernent toutes ses composantes, et sur ses perspectives d'avenir.

Comme ce fut le cas récemment ${ }^{1}$, la Revue a voulu associer des dirigeants et spécialistes de Sociétés nationales, du Secrétariat de la Ligue et du CICR à l'établissement de ce dossier qui n'a pas la prétention d'être exhaustif, mais qui, nous l'espérons, permettra au lecteur, a travers les réflexions, les exemples et les suggestions présentés dans les diverses contributions, de mieux prendre la mesure de ce qu'on peut considérer comme une véritable stratégie de la communication au sein du Mouvement.

Dans une première partie consacrée à la problématique de la communication dans le monde moderne, Sylvie Boiton-Malherbe, professeur de droit de la communication, examine le rôle de la communication, en tant que distribution des connaissances; elle souligne non seulement les atouts favorisant, avec l'appui de la technologie, une plus large circulation des informations et leur effet multiplicateur ainsi que le respect des identités culturelles, mais aussi les effets pervers qu' elle peut provoquer et qui sont autant de facteurs d'inégalités et de tensions.

L'auteur milite dès lors en faveur d'un véritable partage des connaissances et des techniques d'information entre pays développés et pays en développement, qui requiert la concertation, la capacité d'écoute, l'authenticité dans l'échange, l'adéquation des connaissances aux diverses cultures, tant il est vrai que seules l'éducation et la formation pourront contribuer à des changements de mentalité et favoriser le dialogue (voir p. 193).

Concertation, coopération, dialogue sont aussi les mots clés de la Politique d'information du Mouvement qu'analysent conjointement,

1 Voir RICR, $\mathrm{X}^{\mathrm{e}}$ anniversaire de l'adoption des Protocoles additionnels aux Conventions de Genève (1977-1987), No.765, mai-juin 1987; Développement et coopération au sein du Mouvement international de la Croix-Rouge et du Croissant-Rouge, No. 771, mai-juin 1988. 
dans une deuxième partie, Michèle Mercier et George Reid, respectivement responsables de la communication au CICR et au Secrétariat de la Ligue (voir p. 208). Cette politique qui repose sur une Déclaration d'identité, fortement ancrée dans les Statuts et les Principes fondamentaux du Mouvement et sur un Programme d'identité vise à renforcer la capacité du Mouvement à communiquer de façon précise et efficace ce qu'il est, ce qu'il fait et pourquoi il le fait, tout en mettant l'accent sur la spécificité du Mouvement.

Comme le soulignent les auteurs, les mots doivent se traduire par des actes, la politique d'information doit être un véritable programme d'action, intégré dans les activités quotidiennes des composantes du Mouvement. Elle doit permettre aux Sociétés nationales, au CICR et à la Ligue de s'identifier chacun selon les circonstances tout en servant le même but: créer les conditions nécessaires pour atteindre les victimes et alléger les souffrances humaines.

$$
* * *
$$

Quel est le rôle de la communication dans le développement et la promotion des activités humanitaires des Sociétés nationales de la Croix-Rouge et du Croissant-Rouge? Pour répondre à cette question qui constitue la troisième partie de ce dossier, la Revue a invité plusieurs Sociétés nationales des différentes régions du monde à examiner et évaluer comment les relations interpersonnelles, entre leurs volontaires et les communautés, par exemple, et les moyens d'information dont elles disposent contribuent à promouvoir leurs activités humanitaires. Quelles ont été les leçons de leurs expériences, quels ont été les obstacles rencontrés et les moyens de les surmonter? Comment entendent-elles mettre en auvre le Programme d'identité du Mouvement afin de renforcer leur propre politique d'information?

Les Sociétés nationales de la Croix-Rouge ou du Croissant-Rouge de Bulgarie, d'El Salvador, des Etats-Unis d'Amérique, du Malawi, du Maroc, des Pays-Bas et des Philippines, à travers leurs contributions (voir pp. 219), expliquent comment leurs services d'information ou l'action de leurs volontaires dans leurs contacts quotidiens avec les communautés contribuent, ici à promouvoir la diffusion du droit international humanitaire et des principes du Mouvement, là à développer les services à la communauté, dans les zones rurales et urbaines, là encore à servir de support au recrutement de membres et à la recherche de fonds. Des éclairages intéressants sont apportés sur l'importance du Programme d'identité, sur les plans national et international, pour recueillir l'adhésion du grand public et sur les effets 
bénéfiques de la collaboration entre les Sociétés nationales et les médias de leurs pays.

Ces articles, à travers les nombreux exemples présentés, ont le mérite d'exposer les problèmes auxquels les Sociétés nationales sont confrontées, qu'il s'agisse de faiblesses structurelles et du manque de ressources, ou bien des effets de la multiplication des organisations humanitaires, préjudiciables parfois à la cohérence de l'image du Mouvement. Tous les auteurs, à des degrés divers, sont conscients qu'il ne peut y avoir de véritable développement des activités des Sociétés nationales qui ne repose sur une politique d'information cohérente et rigoureusement définie.

$$
* * *
$$

La quatrième partie de ce dossier, axée sur l'avenir, est consacrée au programme de soutien aux Sociétés nationales en développement dans le domaine de l'information (voir p. 273). Helena Korhonen, chef des programmes de développement à la Croix-Rouge finlandaise, rappelle que l'information n'est pas une fin en soi, mais qu'elle doit être avant tout un service d'appui, un moyen de promouvoir les principes et les activités de la Croix-Rouge et du Croissant-Rouge et qu'elle est donc partie intégrante du développement. Tirant les leçons d'une expérience personnelle en Afrique et évaluant l'aide au développement des Sociétés nationales, l'auteur dégage les grandes lignes d'un Programme de soutien aux Sociétés en développement en matière d'information, l'accent étant mis sur l'aide à la planification et au financement des services d'information, à la fourniture de matériel adapté et surtout à la formation des communicateurs. Un tel programme implique un véritable partenariat entre Sociétés opératrices et Sociétés participantes reposant sur le partage des connaissances et des techniques, sur le respect des cultures et traditions et sur la volonté d'engagement à long terme.

\section{La Revue}

